\title{
Estudio longitudinal del estado nutricional y de la condición física de estudiantes de enseñanza media de un liceo municipal de Talca. Chile
}

\author{
Longitudinal study of the nutritional status and physical condition of high school \\ students of a Municipal High School of Talca. Chile \\ * Patricio Zúñiga C., ** Abner Jaque B., ** Dorys González P., *** Cesar Faúndez C.
}

Zuñiga, P., Jaque, A., González, D., \& Faúndez, C. (2018). Estudio longitudinal del estado nutricional y de la condición física de estudiantes de enseñanza media de un liceo municipal de Talca. Chile. Revista Ciencias de la Actividad Física $U C M, \mathrm{~N}^{\circ} 19$ (2) julio-diciembre, 21-30. DOI: http://doi.org/10.29035/rcaf.19.2.4

\section{RESUMEN}

El objetivo de esta investigación es analizar las variables de estado nutricional y condición física de estudiantes de dos niveles de enseñanza media (primero y cuarto) durante los años 2016, 2017 y 2018, para saber cómo ingresan y cómo egresan del establecimiento. Se evaluaron parámetros de peso, talla y perímetro de cintura para el estado nutricional, mientras que para la condición física se contemplaron pruebas físicas de salto horizontal y flexo-extensión de codos. Las variables peso y salto horizontal sufrieron cambios. La primera tuvo un aumento constante durante los años de estudio en Primer Año Medio $(\mathrm{p}<0,068)$, lo cual conllevó a un aumento del IMC $(\mathrm{p}<0,054)$ mientras el salto horizontal tuvo una disminución significativa en Cuarto Año Medio $(\mathrm{p}<0,004)$. Con esto se determina que los parámetros de estado nutricional y condición física de los estudiantes evaluados sufren efectos negativos durante los años de investigación.

\section{PALABRAS CLAVE}

Estado nutricional, condición física, obesidad, adolescentes.

\begin{abstract}
The objective of this research is to analyze the variables or nutritional status and physical condition of students from two high school grades (first and fourth year) during the years 2016, 2017 and 2018, to know their measurements upon entering and when leaving the establishment. For this, parameters of weight, height and waist circumference were evaluated in terms of nutritional status, while for physical condition; physical tests such as horizontal jump and elbow flexionextension were contemplated. The variables of weight and horizontal jumps underwent changes. The first had a constant increase during the years of study in first year $(\mathrm{p}<0,068)$, which led to an increase in BMI $(\mathrm{p}<0,054)$ while the horizontal jump had a significant decrease in fourth year $(\mathrm{p}<0,004)$. This determines that the parameters of nutritional status and physical condition of the students evaluated suffered negative effects during this research.
\end{abstract}

Key words

Nutritional status, physical condition, obesity, adolescents.

* Escuela de Educación Física Universidad Católica del Maule, Chile.

** Liceo Industrial de Talca, Chile.

*** Departamento de Ciencias de la Actividad Física Facultad de Educación UCM, Chile. 
Zuñiga, P., Jaque, A., González, D., \& Faúndez, C. (2018). Estudio longitudinal del estado nutricional y de la condición física de estudiantes de enseñanza media de un liceo municipal de Talca. Chile. Revista Ciencias de la Actividad Física UCM, N 19(2) julio-diciembre, 21-30. ISSN: 0719-4013.

DOI: http://doi.org/10.29035/rcaf.19.2.4

\section{INTRODUCCIÓN}

Dentro de los problemas de salud más presentes en la actualidad a nivel mundial y nacional se encuentra la obesidad, el cual no solo afecta a adultos, sino que también a niños y adolescentes (Ortega, Ruiz \& Castillo, 2013, Díaz, Mena, Chavarría, Rodríguez \& Valdivia-Moral, 2013). Este problema se relaciona principalmente con la disminución de la actividad física y un estilo de vida sedentario, lo que tiene una asociación directa con el estatus nutricional de la persona (Bazán, Santa María \& Laiño, 2014, Gálvez, Rosa, García-Cantó, Rodríguez, Pérez-Soto, Tarraga, \& Tarraga López, 2015). Esta enfermedad consiste, según menciona la OMS, en la acumulación anormal de grasa que es perjudicial para la salud (OMS, 2017). Dicha condición ha ido en aumento en las últimas décadas (Ng et al., 2014) y puede estimarse a través de distintos criterios y/o evaluaciones como las de perímetros, pliegues cutáneos, índice de masa corporal, índice cintura-cadera e porcentaje de grasa, entre los más conocidos.

La etapa de la adolescencia es una etapa vulnerable (Isla, Bernal, Pankow, Rivarola, Villalba, \& de Arbo, 2015) y decisiva de la vida, ya que tiene directa relación con la adquisición de hábitos, debido a que en este periodo el adolescente va madurando y adoptando los modelos que la sociedad le muestre y que para él resulten atractivos e influyentes (Gutiérrez, Aldea, del Mar Cavia, \& Alonso-Torre, 2015). Justamente la adquisición de hábitos poco saludables como la malnutrición, que es una patología dada por el desequilibrio del estado nutricional (Medina, Vargas, Ibáñez \& Rodríguez, 2015) y/o sedentarismo, son los que ocasionan problemas de peso, los cuales resultan perjudiciales en esta etapa, ya que, si se tiene sobrepeso u obesidad en la infancia o adolescencia, esto, se asociará fuertemente con sobrepeso u obesidad en la edad adulta, (Ortega et al., 2013) por lo que la actividad física se vuelve fundamental para promover un desarrollo saludable en las etapas de infancia y adolescencia (Oviedo, Sánchez, Castro, Calvo, Sevilla, Iglesias \& Guerra, 2013). Asimismo, es importante señalar que esta inactividad física contribuye a un factor de riesgo hacia enfermedades crónicas no transmisibles como en- fermedades cardiovasculares, diabetes tipo II y la misma obesidad (Sánchez, Jardón, Rodríguez \& Domínguez, 2014), por lo que su importancia se vuelve aún mayor.

La encuesta nacional de salud de los años 2016-2017 indica que, de los jóvenes de entre 15 y 19 años de edad, el 40,8\% tiene problemas nutricionales relacionados con IMC; del total de jóvenes encuestados, $27,6 \%$ tiene sobrepeso, $12,2 \%$ obesidad, contra un $54 \%$ con condiciones normopeso. Esto se relaciona con los mismos índices de sedentarismo expuestos en esta encuesta, donde el 73,5\% de los encuestados no practica actividad física con una frecuencia mínima de 3 veces por semana (MINSAL, 2017).

En Chile se manifiesta un aumento de la obesidad a medida que aumenta la edad escolar (Martínez, Reinike, Silva, Carrasco, Collipal \& Jiménez, 2013, Murga, 2013). Respecto a la condición física, si bien existen estudios que mencionan que a medida que avanza la edad la condición física es mejor (Barrueco, Buces del Castillo, González, Velasco, López \& Marrodán, 2016), existen otros estudios en que los niveles de actividad física en jóvenes disminuye conforme avanza la edad (Yáñez, Hespanhol, Gómez, Cossio-Bolaños, 2014). Es por esta razón que se vuelve de vital importancia trabajar este ámbito desde la infancia y continuar con dicho trabajo durante la adolescencia, de tal modo que se creen los hábitos saludables correspondientes y se genere conciencia de lo importante que es la actividad física en nuestras vidas y en nuestra salud.

Actualmente, la información procedente de estudios longitudinales que miden objetivamente la actividad física en niños/adolescentes es escasa, pero a la vez consistente. Esta información demuestra que la prevalencia de sobrepeso en escolares adolescentes es alta, lo que podría deberse entre otras cosas, al bajo nivel de actividad física que tiene el alumnado y que se remite solo a la actividad realizada en las clases de educación física (Díaz et al., 2013).

Detectar aquellos sujetos que tienen sobrepeso u obesidad y observar la relación que aquello tiene con el nivel de condición física puede ser importante para evitar problemas 
de salud a medio o largo plazo (Gálvez, Rodríguez, Rosa, García, Pérez, Tárraga Marcos, \& Tárraga López, 2015) y de esta manera fortalecer estrategias preventivas ante la emergencia de la obesidad (Isla et al., 2015).

\section{METODOLOGÍA}

El objetivo de esta investigación fue analizar longitudinalmente el estado nutricional y la condición física de estudiantes de Primer y Cuarto Año de Enseñanza Media de un liceo municipal de la ciudad de Talca durante los años 2016, 2017 y 2018.

Se desarrolló un estudio descriptivo longitudinal de corte transversal, en el que se comparó tres mediciones transversales efectuadas a estudiantes que cursaron Primero y Cuarto Año Medio en los años 2016, 2017 y 2018. La muestra del 2016 se constituyó de 162 estudiantes, la del 2017, por 85, y la del 2018, por 119. Todas las muestras provienen de un establecimiento municipalizado de la ciudad de Talca, Chile. Los alumnos en todas las cohortes fueron seleccionados de forma no-probabilística (accidental), no aleatoria y por conveniencia (Otzen \& Manterola, 2017).

Este estudio además se llevó a cabo siguiendo las normas éticas concordantes en la declaración de Helsinki referentes al anonimato de los nombres y la confidencialidad de los datos obtenidos en investigaciones con seres humanos. Se incluyó en todas las cohortes a los estudiantes que estaban comprendidos entre los 13 a 20 años y a los que autorizaron el consentimiento informado. Fueron excluidos los que presentaban problemas físico- motores que impedían la valoración antropométrica y a los que no asistieron el día de la evaluación, así también como los estudiantes pertenecientes a Segundo Año Medio y Tercer Año Medio, junto con los que tuvieron alguna evaluación de condición física y/o estado nutricional sin registrar.

Se informó previamente al establecimiento respecto al objetivo de este estudio y cómo influiría en él. Se realizó una solicitud formal a través de una carta, entregada a la directora del establecimiento para trabajar con los datos que han sido recolectados en los años académicos 2016-2017-2018 por los profesores de la asignatura de educación física respecto al estado nutricional (IMC y perímetro de cintura) y condición física (salto largo a pies juntos, flexo-extensiones de codos).

La recolección de información se llevó a cabo durante todo el mes de marzo en el respectivo año académico cursado. El peso corporal fue medido con una balanza con precisión de $0,1 \mathrm{~kg}$. La talla fue medida utilizando un estadiómetro con precisión de $0,1 \mathrm{~cm}$. El perímetro de cintura fue medido con una cinta métrica con precisión de $0,1 \mathrm{~cm}$. Estas variables fueron medidas por profesionales del área de educación física pertenecientes al establecimiento en conjunto con alumnos en práctica. Con los resultados de peso y talla se pudo estimar el índice de masa corporal $(\mathrm{kg} /$ $\mathrm{m}^{2}$ ) a través de la relación del peso corporal con la estatura al cuadrado (Vargas, Bahamonde, Cancino, Correa, Michelow, Gatica, Gómez, Martínez, Vargas, Luna, Cossio-Bolaños, 2014) y una vez calculado el resultado del IMC se procedió a clasificar a los estudiantes según dicho resultado. Para esto se utilizó la clasificación de FANTA Project para estudiantes menores de 18 años, la cual clasifica los según "desnutrición severa, desnutrición moderada, normal, sobrepeso, obesidad", dependiendo de la edad decimal del sujeto. Además, se realizaron pruebas físicas para evaluar su condición física mediante pruebas de salto largo a pies juntos y flexo-extensiones de codos durante 60 segundos.

Para establecer la normalidad de la distribución de los datos se utilizó la prueba de Kolmogorov-Smirnov (K-S) y se aplicó a todas las variables, aunque por separado para cada sexo y año de evaluación. Se analizaron los datos por medio de estadística descriptiva de media aritmética y desviación estándar. Para determinar las diferencias significativas entre las cohortes 2016, 2017 y 2018, se utilizó por medio de ANOVA de un factor para medidas independientes. En todos los casos se adoptó diferencias significativas de $\mathrm{p}<0,05$. Todos los cálculos fueron efectuados en planillas de Excel y SPSS 18.0. 


\section{RESULTADOS}

Las características de la muestra de los escolares se observan en la Tabla 1 . El $44,26 \%$ eran alumnos de $2016,23,22 \%$ de 2017 y $32,51 \%$ de 2018.

\section{Tabla 1}

Características de la muestra estudiada según el año de estudio.

\begin{tabular}{ccc}
\hline Año & $\mathrm{N}$ & $\%$ \\
\hline 2016 & 162 & 44,26 \\
\hline 2017 & 85 & 23,22 \\
\hline 2018 & 119 & 32,51 \\
\hline
\end{tabular}

$\mathrm{N}=$ Número de estudiantes; $\%$ = porcentaje.
En la Tabla 2 se observan los valores medios y desviación estándar de las características generales (edad, peso y estatura), durante los años 2016, 2017 y 2018. En cuanto a la estatura y edad de los alumnos de Primer Año Medio, no hubo diferencias estadísticamente significativas. Sin embargo, los estudiantes de ingreso 2018 en Primer Año Medio muestran mayor peso que aquellos estudiantes de ingreso 2016 ( $p<0,068$ ), lo cual representa una tendencia hacia el aumento de peso con el paso de los años, reflejado también en un aumento en el año 2017 y posteriormente un aumento mayor en el año 2018. Respecto a las características de Cuarto Año Medio, la variable de peso se mantiene sin modificaciones al igual que las variables de estatura y edad, las cuales no representan una diferencia estadísticamente significativa.

Tabla 2

Variables antropométricas que caracterizan a la muestra estudiada según año de estudio.

\begin{tabular}{|c|c|c|c|c|c|c|c|}
\hline \multirow{2}{*}{ Indicadores } & \multicolumn{2}{|c|}{2016} & \multicolumn{2}{|c|}{2017} & \multicolumn{2}{|c|}{2018} & \multirow{2}{*}{ P-Valor } \\
\hline & $\mathrm{X}$ & $\mathrm{DE}$ & $\mathrm{X}$ & $\mathrm{DE}$ & $\mathrm{X}$ & $\mathrm{DE}$ & \\
\hline \multicolumn{8}{|c|}{ Primero Medio } \\
\hline Edad & 14,84 &, 8165 & 14,72 & ,7642 & 14,73 & ,9043 & 0,507 \\
\hline Peso & 64,01 & 14,291 & 65,19 & 14,569 & 67,36 & 14,541 & ${ }^{\star} 0,068$ \\
\hline Estatura & 166,24 & 7,824 & 165,97 & 8,363 & 166,73 & 7,342 & 0,81 \\
\hline \multicolumn{8}{|c|}{ Cuarto Medio } \\
\hline Edad & 17,6098 & 0,68775 & 17,88 & 0,71808 & 17,79 & 0,73645 & 0,346 \\
\hline Peso & 75,05 & 14,459 & 73,5 & 14,468 & 75,05 & 22,663 & 0,945 \\
\hline Estatura & 175,05 & 8,794 & 171,94 & 7,81 & 171,14 & 7,143 & 0,166 \\
\hline
\end{tabular}

$\mathrm{X}=$ Promedio; $\mathrm{DE}=$ desviación estándar; ${ }^{*}$ diferencias significativa $\mathrm{p}<0,05$.

En la Tabla 3 se observan los valores medios y desviación estándar del estado nutricional de los estudiantes según el año de estudio, medida a través del IMC (índice de masa corporal) y PC (perímetro de cintura). Los alumnos de Primero Medio mostraron una tendencia hacia el alza en su valor de IMC $(\mathrm{p}<0,054)$ con el transcurso de los años y una tendencia hacia la baja en cuanto al PC $(\mathrm{p}<0,059)$, mientras tanto, en Cuarto Medio no hubo diferencias significativas en IMC ni en PC, a pesar de que en este último hubo un descenso en su valor pero que no es considerado como estadísticamente significativo $(\mathrm{p}<0,656)$. 
Tabla 3

Valores medios y DS de estado nutricional de la muestra estudiada según año de estudio.

\begin{tabular}{|c|c|c|c|c|c|c|c|}
\hline \multirow{2}{*}{ Indicadores } & \multicolumn{2}{|c|}{2016} & \multicolumn{2}{|c|}{2017} & \multicolumn{2}{|c|}{2018} & \multirow{2}{*}{ P-Valor } \\
\hline & $\mathrm{X}$ & $\mathrm{DE}$ & $\mathrm{X}$ & $\mathrm{DE}$ & X & $\mathrm{DE}$ & \\
\hline \multicolumn{8}{|c|}{ Primero Medio } \\
\hline IMC & 23,02 & 4,38685 & 23,86 & 4,97998 & 24,57 & 5,01969 & ${ }^{\star} 0,054$ \\
\hline $\mathrm{PC}$ & 83,19 & 12,627 & 79,28 & 12,363 & 80,15 & 11,484 & ${ }^{*} 0,059$ \\
\hline \multicolumn{8}{|c|}{ Cuarto Medio } \\
\hline IMC & 24,41 & 3,86956 & 25,29 & 4,60336 & 25,6 & 6,70167 & 0,643 \\
\hline PC & 85,68 & 11,252 & 83,67 & 13,359 & 82,62 & 14,386 & 0,656 \\
\hline
\end{tabular}

$\mathrm{IMC}=$ índice de masa corporal; $\mathrm{PC}=$ perímetro de cintura; ${ }^{*}$ diferencias significativa $\mathrm{p}<0,05$.

En la Tabla 4 se observan resultados acerca de un análisis de frecuencia sobre la clasificación del índice de masa corporal de los estudiantes según el año de estudio y nivel de escolaridad. El grupo con IMC "normal” ha ido descendiendo año tras año, en que su mayor porcentaje, en los años 2016 y 2017 , se encontraba en los estudiantes de Cuarto Medio, pero en el año 2018 el grupo de estudiantes con
IMC "normal” fue el de Primero Medio. Con el paso de los años los estudiantes con normopeso fueron menos, los cuales pasaron a aumentar su IMC, agrupándose en las categorías de Sobrepeso y Obesidad. En el último año de evaluación los estudiantes con sobrepeso y obesidad fueron más que en los años anteriores en ambos niveles de escolaridad.

\section{Tabla 4}

Valores de frecuencia de clasificación de índice de masa corporal de los estudiantes según año de estudio.

\begin{tabular}{lcccccc}
\hline \multirow{2}{*}{ Categorías } & \multicolumn{2}{c}{2016} & \multicolumn{2}{c}{2017} & \multicolumn{2}{c}{2018} \\
\cline { 2 - 7 } & $1^{\circ}$ & $4^{\circ}$ & $1^{\circ}$ & $4^{\circ}$ & $1^{\circ}$ & $4^{\circ}$ \\
\hline D.M & $8,0 \%$ & $0,0 \%$ & $0,0 \%$ & $0,0 \%$ & $0,0 \%$ & $9,5 \%$ \\
\hline Normal & $50,4 \%$ & $59,5 \%$ & $49,3 \%$ & $50,0 \%$ & $37,8 \%$ & $33,3 \%$ \\
\hline Sobrepeso & $24,0 \%$ & $24,3 \%$ & $25,4 \%$ & $22,2 \%$ & $32,7 \%$ & $28,6 \%$ \\
\hline Obesidad & $24,8 \%$ & $16,2 \%$ & $25,4 \%$ & $27,8 \%$ & $29,6 \%$ & $28,6 \%$ \\
\hline Total & $100 \%$ & $100 \%$ & $100 \%$ & $100 \%$ & $100 \%$ & $100 \%$ \\
\hline
\end{tabular}

D.M= Desnutrición moderada; $1^{\circ}=$ Primero medio; $4^{\circ}=$ Cuarto Año Medio.

Los valores medios y desviación estándar de la condición física, medida a través de fuerza de brazos y piernas durante los años 2016, 2017 y 2018, están presentes en la Tabla 4. Los datos obtenidos en los estudiantes de Primero Medio muestran que existen diferencias significativas respecto a la fuerza de brazos medida a través de flexo-extensiones de codos $(\mathrm{p}<0,009)$, mientras que en la fuerza de tren inferior medida a través de salto horizontal existen cambios no significativos $(\mathrm{p}<0,068)$. Sin embargo, dicha diferencia no sigue un patrón hacia el aumento o hacia la baja con el paso de los años. Entre tanto, en los resultados obtenidos de los alumnos de Cuarto Medio, la variable de fuerza de tren superior en 2017 aumenta en relación a la fuerza de tren superior que tuvieron los estudiantes en el año 2016, pero en el año 2018 no sigue la misma tendencia, sino que disminuye en comparación al año anterior, aunque sigue siendo mayor que en el primer año de evaluación, por lo tanto, no es un dato significativo. Por otra parte, en la fuerza de tren inferior de este nivel sí existe una diferencia estadísticamente significativa ya que cada año su valor es menor $(p<0,004)$. 
Zuñiga, P., Jaque, A., González, D., \& Faúndez, C. (2018). Estudio longitudinal del estado nutricional y de la condición física de estudiantes de enseñanza media de un liceo municipal de Talca. Chile. Revista Ciencias de la Actividad Física UCM, N 19(2) julio-diciembre, 21-30. ISSN: 0719-4013.

DOI: http://doi.org/10.29035/rcaf.19.2.4

Tabla 5

Valores Medios y DE sobre la condición física de la muestra estudiada según año de estudio.

\begin{tabular}{|c|c|c|c|c|c|c|c|}
\hline \multirow{2}{*}{ Indicadores } & \multicolumn{2}{|c|}{2016} & \multicolumn{2}{|c|}{2017} & \multicolumn{2}{|c|}{2018} & \multirow{2}{*}{ P-Valor } \\
\hline & $\mathrm{X}$ & $\mathrm{DE}$ & $\mathrm{X}$ & $\mathrm{DE}$ & $\mathrm{X}$ & $\mathrm{DE}$ & \\
\hline \multicolumn{8}{|l|}{ Primero Medio } \\
\hline F. Brazos & 27,25 & 13,667 & 21,73 & 6,3 & 26,95 & 14,016 & ${ }^{\star} 0,009$ \\
\hline F. Piernas & 163,55 & 28,627 & 169,42 & 32,235 & 158,72 & 27,055 & ${ }^{\star} 0,068$ \\
\hline \multicolumn{8}{|l|}{ Cuarto Medio } \\
\hline F. Brazos & 27,22 & 9,744 & 34,06 & 12,767 & 30,81 & 15,763 & 0,150 \\
\hline F. Piernas & 191,54 & 23,816 & 186,44 & 28,036 & 165,57 & 33,979 & ${ }^{\star} 0,004$ \\
\hline
\end{tabular}

F. Brazos $=$ flexo-extensiones de codo; F. Piernas $=$ Salto horizontal a pies juntos;

*diferencias significativa $\mathrm{p}<0,05$

\section{DISCUSIÓN}

El peso de los estudiantes fue uno de los parámetros que se modificó en los estudiantes que ingresaban a Primero Medio al establecimiento estudiado. Este parámetro fue en aumento cada año, lo que se confirma al comparar los datos de estudiantes que ingresaron a Primero Medio en el año 2016, con aquellos que ingresaron en el año 2017, y con los del año 2018. Además, si se compara el peso de los estudiantes del mismo año de Primero Medio vs Cuarto Medio, se observa que existe un aumento considerable de peso, pero si se compara el peso de los estudiantes de Cuarto Medio en los diferentes años, no se observa una diferencia significativa, sino que se mantiene constante. En el estudio de Murga se encontraron resultados similares, ya que el peso aumentó considerablemente de una evaluación a otra (Murga, 2013).

Las variables de estado nutricional como IMC y PC obtuvieron cambios importantes en los estudiantes de Primero Medio. La primera variable fue en aumento año tras año, mientras que la segunda fue en disminución. Los estudiantes con sobrepeso y obesidad fueron más en el año 2018 en ambos niveles de escolaridad (Primero y Cuarto Medio), al mismo tiempo que aquellos con IMC normal se agruparon en porcentajes menores en comparación con el primer año de estudio. En el estudio de Murga, la variable IMC también aumentó, y lo hizo en $1,3 \mathrm{~kg} / \mathrm{m}^{2}$, mientras que en este estudio la diferencia fue de $1,55 \mathrm{~km} / \mathrm{m}^{2}$, es decir, hubo resultados similares. Ahora bien, al comparar los resultados de IMC y PC del mismo año entre estudiantes de Primero y Cuarto Medio, evidencian que los alumnos egresan del establecimiento con mayores niveles de IMC y PC que cuando ingresan. Es importante revisar el significado que entrega el resultado de esta evaluación de IMC, puesto que al ser jóvenes menores de edad se optó por clasificar el IMC en función del Project FANTA, la cual clasifica según edad decimal. Esta clasificación indica que el IMC de los estudiantes de primero con un promedio de edad de 14,84 en 2016 , se encuentran en la categoría de "normopeso". Sin embargo, estudiantes del mismo nivel, pero evaluados en el año 2018 con un promedio de edad de 14,73 se encuentran en la categoría de "sobrepeso", es decir, en 2 años los estudiantes pasaron de estar con IMC normal a tener un IMC con sobrepeso. La tendencia en Cuarto Medio es similar; en 2016 se encontraban en la categoría de "normopeso", pero hasta la fecha de 2018 avanzaron a la categoría de "sobrepeso", debido al descenso en su estatura.

En estudiantes de Cuarto Medio se apreció una disminución significativa $(\mathrm{p}<0,004)$ en cuanto a la fuerza de la musculatura del tren inferior desde 2016 hasta el 2018. En cuanto a los alumnos de Primero Medio solo hubo una tendencia a disminuir en los años en que se realizaron las evaluaciones $(\mathrm{p}<0,068)$. Respecto de la fuerza de tren superior, no se observaron diferencias significativas durante el 
transcurso de los años. Sin embargo, se logra observar una tendencia a mejorar el valor de la fuerza de dicha musculatura en alumnos que egresan respecto de los que ingresan según año. Esta baja en la fuerza de la musculatura de tren inferior puede estar relacionada con el aumento de peso de los estudiantes, ya que a mayor peso, mayor fuerza requieren para realizar el ejercicio evaluado. Por último, al comparar el estado nutricional de los estudiantes, es posible observar que cada año los estudiantes que ingresan y egresan al establecimiento, lo están haciendo con mayores índices de exceso de peso, reflejado en la población con sobrepeso y obesidad, las cuales van en aumento, al mismo tiempo que aquella población con normopeso decae año tras año.

Con los resultados de las pruebas de condición física ocurre una situación semejante a las otras variables estudiadas, puesto que en Cuarto Medio los resultados de las pruebas de salto horizontal y flexo-extensión de codos fueron mayores al compararlos con estudiantes de Primero Medio del mismo año. Además, a diferencia de los resultados del estado nutricional, en esta ocasión sí es posible observar diferencias significativas al comparar los datos según el año de estudio. En los estudiantes de Primero Medio la fuerza del tren superior expresada en las repeticiones que hicieron de flexo-extensiones de codo en 60 segundos muestra que en cada año los resultados fueron diferentes al anterior, pero sin seguir una tendencia lineal hacia el alza o descenso, mientras que la fuerza del tren inferior expresada en los centímetros obtenidos en un salto horizontal a pies juntos tuvo un cambio negativo entre el año 2016 y 2018, en que hubo un descenso mayor desde 2017 al año 2018. Datos similares obtuvo Murga en 2013 al comparar estudiantes de 14 y 15 años en los años 1982/83 y 2008/09, aunque en esta ocasión la prueba consistió en un salto de longitud que parte con una carrera de 20 metros previa al rechazo, por lo cual los resultados son mayores, pero siguen la misma tendencia al incremento. En los estudiantes de Cuarto Medio la diferencia fue más evidente, en especial en el salto horizontal, ya que año tras año saltaron menos centímetros, lo cual indica una baja en la fuerza de dicha musculatura, dato que difiere del obtenido por Murga en su estudio, ya que en la prueba de salto Horizontal los rendimientos se mantuvieron.

Como la variable IMC se obtiene de los datos de peso y estatura, este resultado confirma que existe un aumento de peso que ocasiona un alza en los valores de IMC, puesto que el peso va aumentando en los estudiantes de Primero Medio, mientras que la variable de estatura se mantiene constante, lo que podría significar que el peso extra que los estudiantes adquieren se esté acumulando en otras zonas del cuerpo diferentes a la zona abdominal, ya que los parámetros de $\mathrm{PC}$ descienden. En estudiantes de Cuarto Medio no hubo diferencias significativas, aunque variaron los datos en la misma relación, es decir, el IMC aumentó y el PC descendió.

A diferencia de este estudio, hubo otros que lograron resultados diferentes, en el que la condición física fue en aumento, como el caso de Quierolo et al., (2016), quien observó cómo la aptitud física de los jóvenes incrementó positivamente hasta los 16 años respecto de aquellos con 14 años de edad en pruebas como resistencia muscular abdominal, resistencia aeróbica y salto horizontal. Este dato es relevante para esta y futuras investigaciones, puesto que como señalan Yañez et al., 2014, generalmente el nivel de actividad física durante la adolescencia va disminuyendo conforme la edad avanza.

Un valor que no sufrió modificaciones en ninguno de los niveles fue la estatura. Sin embargo, en el estudio de Murga (2013) este parámetro tuvo cambios significativos en estudiantes de 15 años, con un aumento de $6 \mathrm{~cm}$ en promedio desde 1982/83 a 2008/09. Esta diferencia puede deberse a la gran brecha generacional de este estudio en comparación con el nuestro.

En el estudio de Quierolo et al., (2016) los resultados respecto al salto horizontal fueron diferentes a los de este estudio. Los estudiantes de 16 y 17 años (Cuarto Medio) tuvieron aumentos significativos en las pruebas de salto horizontal (tren inferior) en relación con aquellos de 14 años (Primero Medio), resultados similares a los obtenidos en un estudio 
(Barrueco, Buces del Castillo, González, Velasco, López \& Marrodán, 2016), en que la valoración promedio de centímetros logrados en el salto horizontal aumentó significativamente en estudiantes de 17-18 años, comparado con aquellos de 14-15 años.

\section{CONCLUSIONES}

Tras la monitorización de los estudiantes adolescentes que ingresaban y egresaban de la enseñanza media durante tres años de estudio, se determinó que el estado nutricional y la condición física cada año fue empeorando. El principal problema en el estado nutricional fue el aumento de peso, reflejado en los valores de IMC que aumentaron, existiendo una prevalencia mayor en Primero Medio.

Los resultados obtenidos tras la recopilación de datos en esta investigación dejan en evidencia que el estado nutricional de los jóvenes que ingresan al establecimiento en Primero Medio cada vez se desfavorece más. Sin embargo, en aquellos estudiantes que están por egresar en Cuarto Medio, su estado nutricional no se ve tan desfavorecido, sino que se mantiene en niveles similares, situación que podría justificarse debido al trabajo que se está realizando hoy en día en la asignatura de educación física, puesto que a partir del año 2014 en los cursos de Primero y Segundo Medio se incorporó una hora extra, pasando de 2 a 3 horas pedagógicas, mientras que en los niveles de Tercero y Cuarto medio, que no tenían clases de educación física anteriormente, se incorporó la asignatura con 2 horas pedagógicas. Sin embargo, lo anterior, la condición física se ve desfavorecida con el paso del tiempo. Este resultado sugiere un posible riesgo cardio metabólico adverso en la población escolar estudiada. Por lo tanto, es urgente que los programas de prevención de la obesidad se instituyan dentro de los colegios.

Se requiere seguir investigando sobre el tema ya que son pocas las investigaciones longitudinales existentes sobre el estado nutricional y la condición física de adolescentes. Además, dado que la población estudiada varía año tras año y que las evaluaciones tomadas durante estos años no fueron las mismas, no fue posible agregar variables asociadas a la condición física tales como capacidad aeróbica y flexibilidad, por lo que se recomienda que se tomen en cuenta para futuras investigaciones debido al impacto en la salud de los estudiantes con estas características. También es necesario investigar longitudinalmente con los mismos jóvenes durante todo el período de la investigación, de manera que se identifique en qué año es cuando más modificaciones se producen. 


\section{REFERENCIAS BIBLIOGRÁFICAS}

Barrueco Moro, P., Buces del Castillo, M., González Montero de Espinosa, M., Velasco Algaba, E., López Ejeda, N., \& Marrodán Serrano, M. D. (2016). Análisis semilongitudinal de la condición física en adolescentes madrileños. Arch. med. deporte, 183-192.

Bazán, N. E., Santa María, C. J., \& Laiño, F. A. (2014). Actividad física, comportamiento sedentario y estado nutricional en escolares de la ciudad de Buenos Aires. Actualización en Nutrición, 15(3), 52-58.

Díaz Martínez, X., Mena Bastías, C., Chavarría Sepúlveda, P., Rodríguez Fernández, A., \& Valdivia-Moral, P. Á. (2013). Estado nutricional de escolares según su nivel de actividad física, alimentación y escolaridad de la familia. Revista cubana de salud pública, 39, 640-650.

FOOD AND NUTRITION TECHNICAL ASSITANCE (2013). Tablas de IMC y tablas de IMC para la edad de niños(as) $\mathrm{y}$ adolescentes de 5 a 18 años de edad y tablas de IMC para adultos(as) no embarazadas, no lactantes $>19$ años de edad. Recuperado de: https://www.fantaproject.org/sites/default/files/resources/FANTA-BMI-charts-Enero2013ESPANOL 0.pdf

Gálvez Casas, A., Rodríguez García, P. L., Rosa Guillamón, A., García-Cantó, E., Pérez Soto, J. J., Tárraga Marcos, M. L., \& Tárraga López, P. J. (2015). Nivel de condición física y su relación con el estatus de peso corporal en escolares. Nutrición hospitalaria, 31(1).

Gálvez Casas, A., Rosa Guillamón, A., García-Cantó, E., Rodríguez García, P. L., Pérez-Soto, J. J., Tarraga Marcos, L., \& Tarraga López, P. (2015). Estado nutricional y calidad de vida relacionada con la salud en escolares del sureste español. Nutrición Hospitalaria, 31(2).
Gutiérrez, R., Aldea, L., del Mar Cavia, M., \& Alonso-Torre, S. R. (2015). Relación entre la composición corporal y la práctica deportiva en adolescentes. Nutrición Hospitalaria, 32(1), 336-345.

Isla, G. M., Bernal, S. S., Pankow, V. S., Rivarola, M. B., Villalba, G. R., \& de Arbo, L. M. (2015). Perfil nutricional de Escolares y Adolescentes en escuelas públicas y privadas, 2013. Pediatría (Asunción), 42(2), 129-133.

Martínez, C., Reinike, O., Silva, H., Carrasco, V., Collipal, E., \& Jiménez, C. (2013). Composición corporal y estado nutricional de una muestra de estudiantes de 9 a 12 años de edad de colegios municipalizados de la comuna de Padre las casas, región de la Araucanía-Chile. International Journal of Morphology, 31(2), 425-431.

Medina, Ó., Vargas, S. L., Ibáñez, É., \& Rodríguez, G. (2015). Estado nutricional antropométrico de los niños y adolescentes de 17 escuelas del área rural del municipio de La Mesa, Cundinamarca, Colombia, 2012. Revista Salud Bosque, $4(1), 19-28$.

MINSAL (2017). Encuesta Nacional de Salud 2016-2017. Ministerio de Salud Chile. Recuperado de: http://www.minsal. cl/wp-content/uploads/2017/11/ENS2016-17_PRIMEROS-RESULTADOS. pdf

Murga Rodríguez, F. (2013). Análisis de la condición física en escolares gallegos de $14 y$ 15 años. Evolución entre las generaciones 1982/83 y 2008/09 (tesis doctoral). Universidad de Santiago de Compostela. 
Ng, M., Fleming, T., Robinson, M., Thomson, B., Graetz, N., Margono, C., ... \& Abraham, J. P. (2014). Global, regional, and national prevalence of overweight and obesity in children and adults during 1980-2013: a systematic analysis for the Global Burden of Disease Study 2013. The lancet, 384(9945), 766-781.

OMS. (2017). Obesidad y sobrepeso. Recuperado de: http://www.who.int/es/newsroom/fact-sheets/detail/obesity-andoverweight.

Ortega, F. B., Ruiz, J. R., \& Castillo, M. J. (2013). Physical activity, physical fitness, and overweight in children and adolescents: evidence from epidemiologic studies. Endocrinología y Nutrición (English Edition), 60(8), 458-469.

Otzen, T., \& Manterola, C. (2017). Técnicas de Muestreo sobre una Población a Estudio. International Journal of Morphology, 35(1), 227-232.

Oviedo, G., Sánchez, J., Castro, R., Calvo, M., Sevilla, J. C., Iglesias, A., \& Guerra, M. (2013). Niveles de actividad física en población adolescente: estudio de caso. Retos: nuevas tendencias en educación física, deporte y recreación, (23), 43-47.

Queirolo Riffo, L., Rojas Jara, K., Puchi Acuña, C., Gómez Campos, R., Mendez Cornejo, J., \& Cossio Bolaños, M. (2016). Estudio longitudinal de la aptitud física de adolescentes mujeres que efectuaban actividad física durante dos veces por semana. Nutrición Clínica y Dietética Hospitalaria, 36(3), 53-58. DOI: $\underline{10.12873 / 363 q u e i r o l o}$
Sánchez, M. E. D., Jardón, D. S., Rodríguez, L., \& Domínguez, V. M. L. (2014). Actividad física y estado nutricional en adolescentes. Una visión de género. Antropo, (31), 39-49.

Vargas, R., Bahamonde, C., Cancino, J., Correa, P., Michelow, S., Gatica, P., Gómez, R., Martínez, C., Vargas, D., Luna, D., Cossio-Bolaños, M. (2014). Parámetros de crecimiento y adiposidad corporal de adolescentes Chilenos a través de la referencia CDC-2000 y CDC-2012: estudio ACECH. Revista Chilena de Nutricion 41(1), 54-60. DOI: $10.4067 /$ $\underline{\text { S0717-75182014000100007 }}$

Yáñez, A., Hespanhol, J., Gómez, R., CossioBolaños, M. (2014). Valoración de la actividad física en adolescentes escolares por medio de cuestionario. Rev. Chil. Nutr, 41(4). http://dx.doi.org/10.4067/ $\underline{\text { S0717-75182014000400003 }}$

\section{Dirección para correspondencia}

\section{César Faúndez Casanova}

Profesor de Educación Física

Magíster en Ciencias de la Actividad Física, Universidad Católica del Maule.

Académico Departamento de Ciencias de la Actividad Física, Facultad de Ciencias de la Educación

Universidad Católica del Maule, Chile.

Contacto:

cfaunez@ucm.cl

Recibido: 06/07/2018

Aceptado: 28/09/2018 